

\title{
Nigrosome 1 Detection at 3T MRI for the Diagnosis of Early-Stage Idiopathic Parkinson Disease: Assessment of Diagnostic Accuracy and Agreement on Imaging Asymmetry and Clinical Laterality
}

\author{
Y. Noh, Y.H. Sung, J. Lee, and (DE.Y. Kim \\ i. O- EBM
}

\begin{abstract}
BACKGROUND AND PURPOSE: In the early stages of idiopathic Parkinson disease, motor symptoms are usually asymmetric. We aimed to assess the feasibility of nigrosome 1 detection at 3T MR imaging to analyze the agreement of its asymmetry and clinical laterality.
\end{abstract}

MATERIALS AND METHODS: High-resolution 3D multiecho imaging was performed at 3T MR imaging in 13 healthy subjects and 24 patients with idiopathic Parkinson disease confirmed by N-3-fluoropropyl-2- $\beta$-carbomethoxy-3- $\beta$-(4-iodophenyl) nortropane $\left({ }^{18} \mathrm{~F}\right.$-FP-CIT) PET. The nigrosome 1 detection findings by using the MR imaging data were rated as "normal," "possibly abnormal," and "abnormal" by 2 independent reviewers. The degree of ${ }^{18} \mathrm{~F}-\mathrm{FP}$-CIT binding was visually assessed in the caudate nucleus and putamen on PET images. Clinical laterality was evaluated by scores of the Unified Parkinson Disease Rating Scale, Part III. Asymmetry of the affected nigrosome 1 and the degree of ${ }^{18} \mathrm{~F}-\mathrm{FP}$-CIT binding were analyzed for agreement with clinical laterality.

RESULTS: The diagnostic sensitivity, specificity, and accuracy of the nigrosome 1 detection at 3 T MR imaging was $100 \%, 84.6 \%$, and $94.6 \%$, respectively. Interrater agreements for the abnormality and asymmetry of nigrosome 1 were excellent ( $\kappa=0.863$ and 0.835 , respectively). In patients with idiopathic Parkinson disease, the agreement of asymmetry between clinical laterality and nigrosome 1 detection was good $(\kappa=0.724)$. The degree of the ${ }^{18}$ F-FP-CIT PET binding showed fair agreement $(\kappa=0.235)$ with clinical laterality.

CONCLUSIONS: The abnormality involving nigrosome 1 can be detected at 3T MR imaging with an accuracy of $94.6 \%$. The clinical laterality is in high concordance with the laterality of the nigrosome 1 detection at $3 \mathrm{~T}(\kappa=0.724)$.

ABBREVIATIONS: DAT $=$ dopamine transporter; ${ }^{18} \mathrm{~F}-\mathrm{FP}-\mathrm{CIT}=\mathrm{N}$-3-fluoropropyl-2- $\beta$-carbomethoxy-3- $\beta$-(4-iodophenyl) nortropane; H\&Y $=$ Hoehn and $\mathrm{Yahr}$ stage; IPD = idiopathic Parkinson disease; MEDIC = multiecho data image combination; UPDRS III = Unified Parkinson Disease Rating Scale, Part III

$\mathbf{N}$ igrosomes are calbindin-poor zones within the substantia nigra pars compacta. ${ }^{1}$ The nigrosomes are primary subregions of the substantia nigra pars compacta where dopaminergic cells are lost in idiopathic Parkinson disease (IPD). Within the nigrosomes, the maximal cell loss occurs in nigrosome 1 , which is the largest subgroup of the nigrosomes. ${ }^{2}$

Received November 24, 2014; accepted after revision April 12, 2015.

From the Departments of Neurology (Y.N., Y.H.S.) and Radiology (E.Y.K.), Gachon University Gil Medical Center, Incheon, Korea; and Department of Electrical and Computer Engineering (Jongho Lee), Seoul National University, Seoul, Korea.

This research was supported by a grant of the Korea Health Technology R\&D Project through the Korea Health Industry Development Institute, funded by the Ministry of Health and Welfare, Republic of Korea (grant No. HI14C1135) and by the Gachon University Gil Medical Center (2013-06).

Paper previously presented at: Annual Meeting of International Society for Magnetic Resonance in Medicine, May 30 to June 5, 2015; Toronto, Ontario, Canada.

Please address correspondence to: Eung Yeop Kim, MD, Department of Radiology, Gachon University Gil Medical Center, 21, Namdong-daero 774 beon-gil, Namdong-gu, Incheon, 21565, South Korea; e-mail: neuroradkim@gmail.com

-- Indicates open access to non-subscribers at www.ajnr.org

EBM Evidence-Based Medicine Level 2.

http://dx.doi.org/10.3174/ajnr.A4412
Recently, a few researchers have tried to visualize the nigrosome 1 area with 7T MR imaging and demonstrated its feasibility as an imaging biomarker for the diagnosis of IPD. ${ }^{3-6}$ The results, however, had a limited clinical utility because of low availability of 7T MR imaging. Hence, several studies have tried to translate nigrosome 1 detection to $3 \mathrm{~T}$ MR imaging, which is widely available. $^{7-9}$ In the previous studies at 3T, however, patients were confirmed as having IPD by clinical assessment, not by dopamine-transporter (DAT) imaging such as N-3-fluoropropyl-2$\beta$-carbomethoxy-3- $\beta$-(4-iodophenyl) nortropane $\left({ }^{18} \mathrm{~F}\right.$-FP-CIT) PET or SPECT, which is commonly used for an early diagnosis of IPD.

In clinical practice, it is more difficult to diagnose an early stage of parkinsonism than an advanced stage. Therefore, a confirmation by an imaging biomarker would be desirable in the diagnosis of a patient in an early stage. If the nigrosome 1 detection at 3T MR imaging is available for the diagnosis of early-stage IPD, it can minimize the need for the DAT PET or SPECT.

In early stages of IPD, motor symptoms are usually asymmetric. ${ }^{10}$ Unilateral or asymmetric symptoms have been suggested to 
correspond to nigrostriatal degeneration in the contralateral hemisphere. ${ }^{11}$ On the basis of this observation, we hypothesized that the asymmetric dopaminergic cell loss in the substantia nigra pars compacta reflects the contralateral symptoms in early-stage IPD, and the cell loss can be visualized by the signal change in the nigrosome 1 area at 3T MR imaging.

In this study, we investigated the feasibility of the nigrosome 1 detection at 3T MR imaging for the diagnosis of patients with early-stage IPD with abnormal findings on DAT PET imaging. ${ }^{12}$ Additionally, we assessed the agreement of asymmetry between the nigrosome 1 detection and patient symptoms, and the asymmetry between the DAT PET imaging and patient symptoms.

\section{MATERIALS AND METHODS}

This study was approved by the institutional review board of the Gachon University, Gil Medical Center. All patients and healthy subjects gave written informed consent.

\section{Participants}

Twenty-four patients with IPD were recruited from the movement disorder clinic at Gachon University Gil Medical Center from March 2013 to July 2014 (mean age, 63.6 \pm 10.97 years; 14 men and 10 women). The clinical diagnosis was based on UK Parkinson's Disease Society Brain Bank Clinical Diagnostic Criteria. ${ }^{13}$ All patients underwent ${ }^{18} \mathrm{~F}$ - FP-CIT PET for the initial diagnosis. The severity of motor symptoms was evaluated by the Hoehn and Yahr (H\&Y) stages ${ }^{14}$ and Unified Parkinson Disease Rating Scale, Part III (UPDRS III). We enrolled only patients with IPD with $\mathrm{H} \& Y$ stages between 1 and 2 (stages 1 and 1.5, 2 patients each; stage 2, 20 patients). The UPDRS III ranged from 6 to 25 (mean, $13.5 \pm 6.19$ ). The exclusion criteria included the following: 1 ) atypical Parkinson disease (progressive supranuclear palsy, multiple systemic atrophy, and corticobasal degeneration);2) patients with dementia; 3) neurologic diseases other than Parkinson disease; 4) other potential causes of parkinsonism such as structural lesions or metabolic derangement; 5) patients with infarction, hemorrhage, tumors, trauma, or severe white matter hyperintensity (deep white matter lesion of $>25 \mathrm{~mm}$ and caps or bands of $>10 \mathrm{~mm})^{15} ; 6$ ) history of taking drugs that are known to cause parkinsonism: antipsychotics, antiemetic drugs such as metoclopramide, calcium-channel blockers such as flunarizine, amiodarone, sodium valproate, lithium; and selective serotonin reuptake inhibitors such as fluoxetine; and 7) MR imaging contraindication due to metal foreign bodies/implants or claustrophobia.

Thirteen age-matched healthy subjects were recruited (mean age, $61.6 \pm 12.29$ years; 4 men and 9 women). They were recruited with the following inclusion criteria: 1 ) age older than 40 years; 2 ) no history of neurologic or psychiatric diseases; 3 ) no family history of movement disorders; and 4) normal cognition without subjective memory impairment (Mini-Mental State Examination score of >26). All participants underwent the Mini-Mental State Examination. The patients were assessed and scanned while medicated. Demographic and clinical characteristics of the participants are summarized in Table 1.
Table 1: Demographic characteristics and imaging findings in study population ${ }^{a}$

\begin{tabular}{lccc}
\hline & \multicolumn{3}{c}{ Healthy Control } \\
& IPD $(\boldsymbol{n}=\mathbf{2 4})$ & $(\boldsymbol{n}=13)$ & $\boldsymbol{P}$ Value \\
\hline Age $(\mathrm{yr})$ & $63.6 \pm 10.97$ & $61.6 \pm 12.29$ & .620 \\
Female sex (\%) & $10(41.7 \%)$ & $9(69.2 \%)$ & .170 \\
MMSE & $27.5(25.0-29.0)$ & $29.0(28.0-29.5)$ & .036 \\
Onset age (yr) & $62.4 \pm 11.59$ & - & \\
Disease duration (mo) & $9.0(3.0-12.0)$ & - & \\
H\&Y scale (1/1.5/2) & $2 / 2 / 20$ & - & \\
UPDRS I & $1.0(0-2.0)$ & - & \\
UPDRS II & $6.75 \pm 2.85$ & - & \\
UPDRS III & $13.5 \pm 6.19$ & - & \\
\hline
\end{tabular}

Note:-MMSE indicates Mini-Mental State Examination; UPDRS, Unified Parkinson Disease Rating Scale, -, not applicable.

${ }^{\text {a }}$ Data are presented as mean \pm SD for normally distributed variables and median (interquartile ranges) for non-normally distributed variables. The $\chi^{2}$ test with Yates continuity correction was used.

\section{MR Image Acquisition}

All participants underwent MR imaging on a 3T scanner with a 32-channel coil (Magnetom Skyra; Siemens, Erlangen, Germany). Whole-brain sagittal 3D MPRAGE imaging was performed first with the following parameters: TR, $1750 \mathrm{~ms}$; TE, $4.32 \mathrm{~ms}$; TI, 920 ms; matrix, $224 \times 222$; FOV, $307 \times 309$; acceleration factor, 2; acquisition time, 3 minutes 35 seconds. Oblique axial 3D multiecho data image combination (MEDIC; proprietary sequence; Siemens) imaging (multi-echo gradient-echo imaging that combines each TE magnitude by the sum of squares) was performed vertical to the longitudinal axis of the midbrain (Fig $1 A$ ). The parameters for MEDIC were as follows: TR, $88 \mathrm{~ms}$; minimum and maximum TE, 11.1 and $66.9 \mathrm{~ms}$, respectively (the number of combined echoes, 6 ; echo spacing, $11.1 \mathrm{~ms}$ ); flip angle, $10^{\circ}$; echo-train length, 6; thickness, $1.5 \mathrm{~mm}$; section number, 20; matrix, $384 \times 384$; FOV, $192 \times 192$ (in-plane resolution, $0.5 \times$ $0.5 \mathrm{~mm}$ ); acceleration factor, 2 ; acquisition time, 4 minutes 45 seconds. Additionally, oblique coronal 3D MEDIC data were acquired parallel to the longitudinal axis of the midbrain (Fig $1 B$ ) by using the same parameters as in oblique axial imaging (Fig 1A).

\section{Visual Rating of Nigrosome 1}

The nigrosome 1 area on the MEDIC images was defined according to the location that was described in the previous pathologic study. ${ }^{2}$ In healthy subjects, the structure appeared symmetrically with slight hyperintensity compared with the adjacent crus cerebri on the MEDIC images (Fig 1). On the oblique axial MEDIC data, evaluation of the nigrosome 1 was performed on 3 sections: an upper section at the lower tip of red nucleus and 2 successive lower sections (Fig 1A). The oblique coronal MEDIC images were also assessed on 3 sections: an anterior section at the anterior tip of red nucleus and the other 2 successive sections posteriorly (Fig $1 B$ ). Both oblique axial and coronal images were displayed side by side by using OsiriX Imaging Software (Version 5.9, http://www. osirix-viewer.com). A neuroradiologist with 11 years' experience (E.Y.K.) and a neurologist (Y.N.) with 4 years' experience independently reviewed anonymized images without any clinical information. We compared the signal intensity of the central portion of nigrosome 1 with that of the white matter lateral to the decussation of the superior cerebellar peduncles. The nigrosome 1 


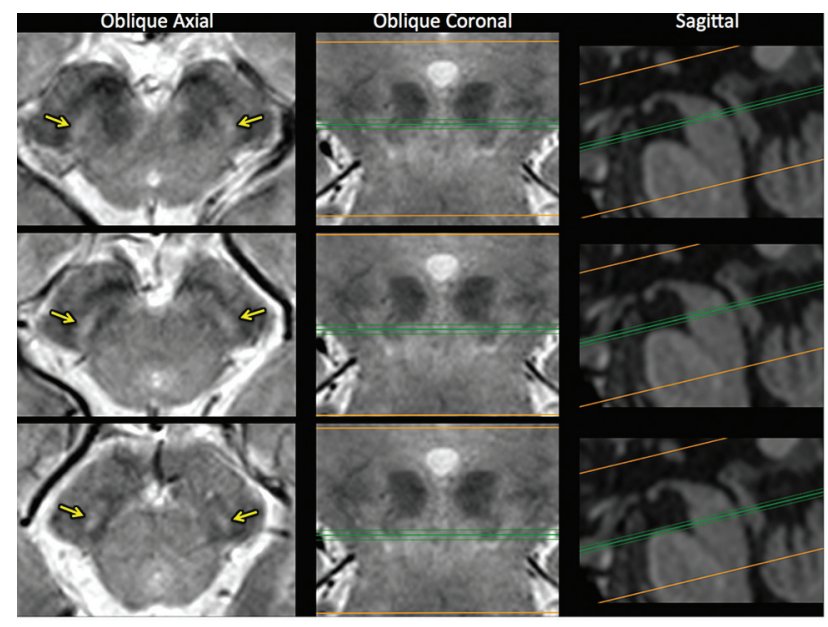

A

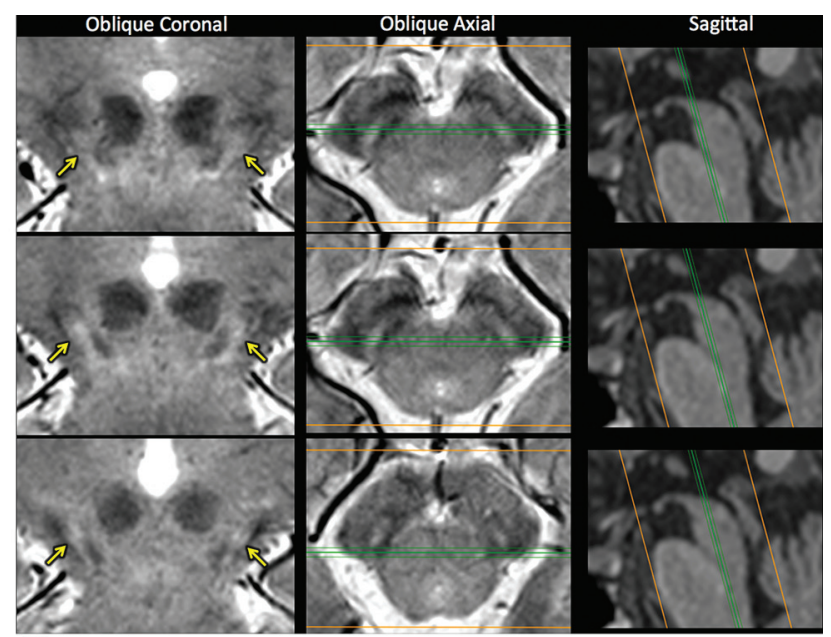

$\mathrm{B}$

FIG 1. Visual assessment of nigrosome 1 in MR imaging. On the oblique axial MEDIC imaging, the evaluation of nigrosome 1 (arrows) was performed on 3 sections: an upper section at the lower tip of the red nucleus and the 2 successive sections (A). The oblique coronal MEDIC imaging was also assessed on 3 sections: an anterior section at the anterior tip of the red nucleus and the 2 consecutive sections (B). In this particular 71-year-old woman, the nigrosome 1 appears normal on both sides.

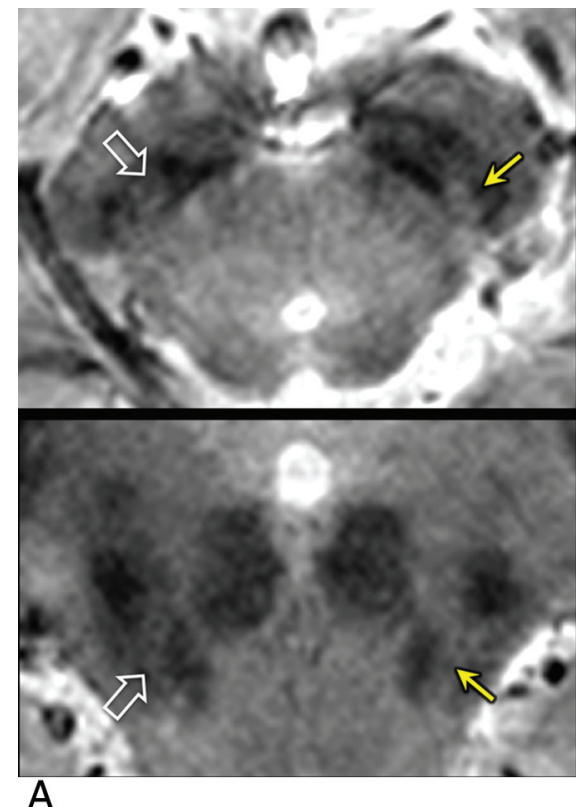

A

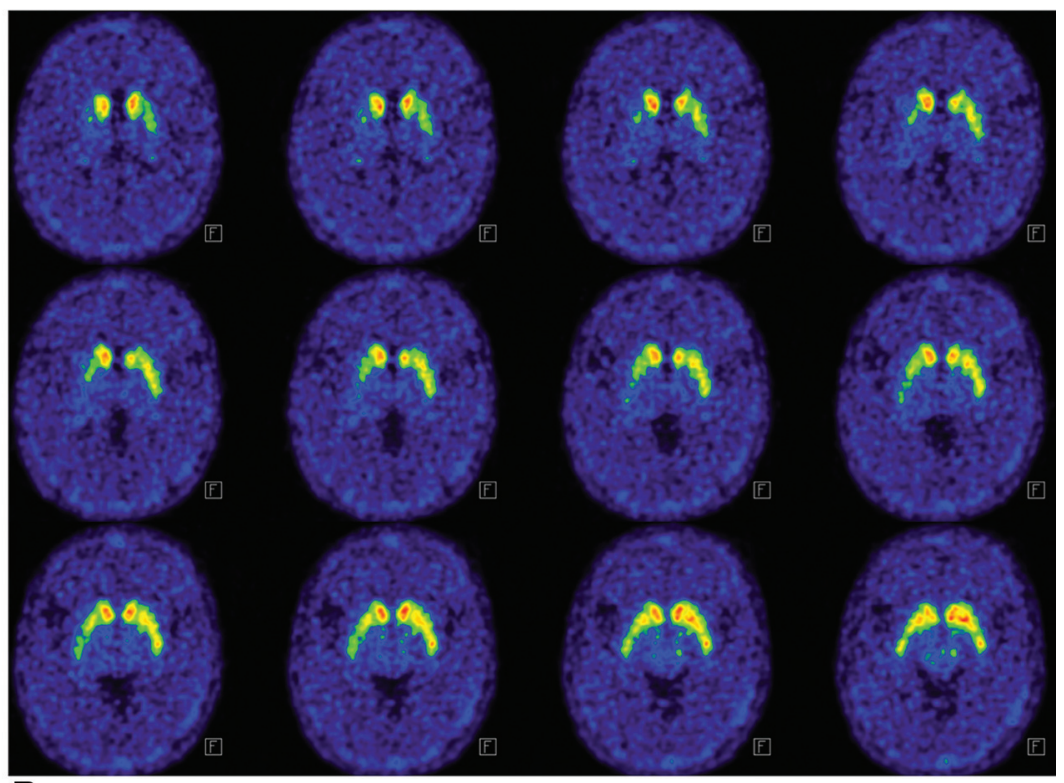

B

FIG 2. Degree of abnormality on MR imaging. Possibly abnormal and definitely abnormal were rated in the left (arrows) and right (open arrows) nigrosome 1, respectively $(A)$. Similar asymmetry was observed on ${ }^{18} \mathrm{~F}$-FP-CIT PET. This 53 -year-old woman has $\mathrm{H} \& \mathrm{Y}$ stage 2 and UPDRS Part III (motor score) of 4 and 1 on the left and right, respectively, which is in concordance with asymmetry on both MR imaging and ${ }^{18} \mathrm{~F}-\mathrm{FP}-\mathrm{CIT}$ PET.

images from the MEDIC imaging were classified into 3 grades: "normal" (iso- or hyperintensity in the central portion of the presumed nigrosome 1) (Fig 1), "possibly abnormal"” (hypointensity in $<50 \%$ of the presumed nigrosome 1) (left nigrosome 1 on Fig 2), and "definitely abnormal" (hypointensity in $\geq 50 \%$ of the presumed nigrosome 1) (right nigrosome 1 on Fig 2). Each side was rated separately. Asymmetry was determined once any difference was rated between the right and left nigrosome 1 . For a simplified statistical analysis, the subjects' images were reclassified as abnormal if any abnormality was determined on either side of the nigrosome 1 area; a subject's image was classified as normal when the bilateral nigrosome 1 was deter- mined to be normal. Any discrepancy between the 2 readers was resolved by consensus.

\section{${ }^{18}$ F-FP-CIT PET Image Acquisition}

All patients with IPD underwent ${ }^{18} \mathrm{~F}$-FP-CIT PET imaging of the brain 120 minutes after injection of 5-mCi (185 MBq) ${ }^{18} \mathrm{~F}-\mathrm{FP}-$ CIT at a PET/CT scanner (Biograph-6; Siemens, Erlangen, Germany). Data were collected in a 3D scanning mode that examined 35 sections (thickness, $4.25 \mathrm{~mm}$ ). Levodopa, dopamine agonist, catechol-O-methyltransferase inhibitors, and monoamine oxidase type B inhibitors were allowed because they are known to have no significant influence on the DAT imaging. ${ }^{16}$ The median 
Table 2: Interpretation of nigrosome 1 by 2 reviewers

\begin{tabular}{lcccccc}
\hline & \multicolumn{2}{c}{ Reviewer 1 } & & \multicolumn{2}{c}{ Reviewer 2 } \\
\cline { 2 - 3 } \cline { 6 - 7 } & Right & Left & & Right & Left \\
\hline Normal (No.) & 10 & 13 & & 12 & 11 \\
Possibly abnormal (No.) & 7 & 5 & & 2 & 3 \\
Definitely abnormal (No.) & 20 & 19 & & 23 & 23 \\
\hline
\end{tabular}

duration between MR imaging and PET was 4.5 months (interquartile range, 2.25-10.75 months).

\section{Visual Rating of ${ }^{18}$ F-FP-CIT PET}

Visual analysis of ${ }^{18} \mathrm{~F}$-FP-CIT binding to the caudate nucleus and putamen was performed by a neurologist (Y.H.S.). No clinical information was provided. Each side was rated separately. The degree of DAT reduction was evaluated by dividing the putamen into anterior and posterior halves along its longitudinal axis. ${ }^{17}$ "Normal" was defined as no discernible reduction of DAT availability in the striatal region (caudate nucleus and putamen). If the reduction of DAT availability was limited in the posterior putamen, the availability was classified as "mild" reduction. When it showed a decrease or absence up to the anterior putamen, it was classified as "moderate" reduction. It was classified as "severe" if the caudate nucleus was involved. ${ }^{18}$ Any difference between right and left DAT availability indicated the presence of asymmetry. No patients with IPD showed normal DAT availability in this study.

\section{Quantification of the Laterality of Symptoms}

The clinical laterality of motor symptoms was evaluated by using the scores of UPDRS III, which was tested bilaterally. Scores of resting tremor, rigidity, finger tapping, hand movement, and rapid alternative movements of hand and leg agility were summed in the right and left sides separately. When the score of one side was higher than that of the other side by 2 points, the symptom was defined as asymmetric.

\section{Statistical Analysis}

Comparisons of demographic and clinical data between patients with IPD and healthy subjects were conducted by the Student $t$ test for normally distributed continuous variables and the MannWhitney $U$ test for non-normally distributed continuous variables. Categoric variables were evaluated by using a $\chi^{2}$ test. Interrater agreement was assessed by using $\kappa$ statistics: weighted $\mathrm{k}$ test for the ratings and the Cohen $\kappa$ test for the 2 groups (normal or abnormal findings). Agreement on the symmetry of MR imaging or PET and clinical laterality was examined by using the Cohen $\kappa$ test. Statistical significance was set at $P<.05$. Statistical analyses were conducted by PASW Statistics 18 (IBM, Armonk, New York) software.

\section{RESULTS}

\section{Diagnostic Accuracy of IPD by Using Nigrosome 1 Detection in MR Imaging}

No scan showed poor image quality or motion artifacts. When the participants were dichotomized as having normal and abnormal findings, 2 of 37 participants were rated differently by the 2 raters. Of the 35 subjects, 26 were rated as having abnormal findings, and 9 subjects were rated as having normal findings (Table 2).

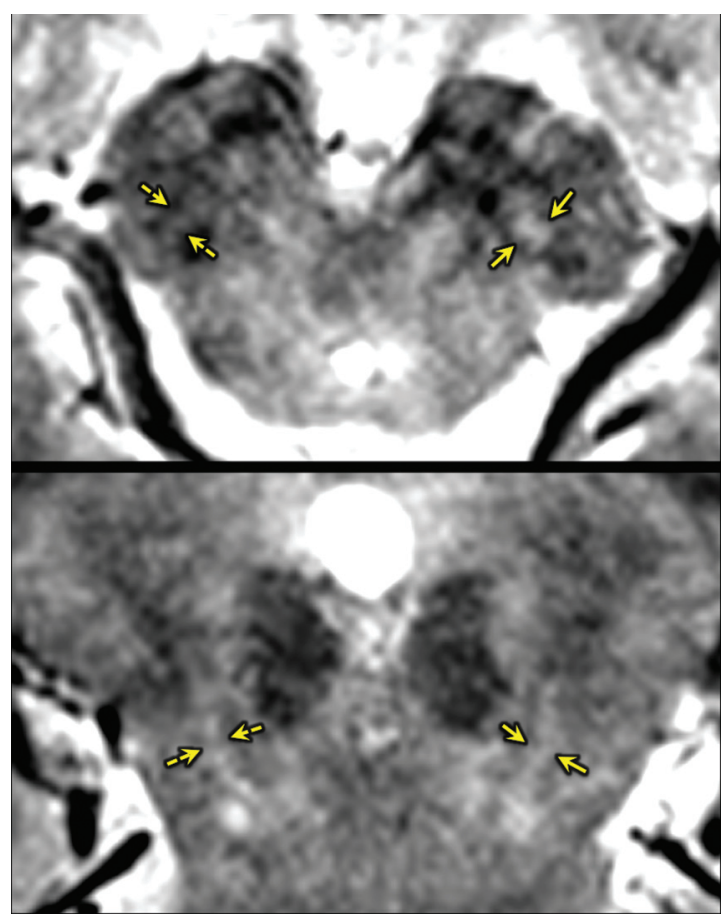

FIG 3. False-positive on MR imaging. In this 64-year-old healthy female subject, both reviewers interpreted the left nigrosome (arrows) was as normal, whereas they called the right one (broken arrows) abnormal.

The interrater agreement on the abnormality of nigrosome 1 findings was excellent $(\kappa=0.863)$. After consensus agreement, the 2 subjects with the rater discrepancies were classified as having normal findings. With the 37 participants, the sensitivity and specificity were $100 \%$ and $84.6 \%$, respectively. The positive predictive value was $92.3 \%(24 / 26)$. The negative predictive value was $100 \%(11 / 11)$. Two of 13 healthy controls were misclassified to the abnormal group (Fig 3). Diagnostic accuracy was $94.6 \%$.

\section{Interrater Agreement on the Laterality of Nigrosome 1}

The interrater agreement on the laterality was excellent $(\kappa=$ 0.835; 95\% CI, 0.673-0.997). For the grading system (normal, possibly abnormal, and definitely abnormal findings), the weighted $\kappa$ value for interrater agreement on the abnormality of the left side was 0.711 (95\% CI, 0.537-0.886), while that of the right side was 0.792 (95\% CI, 0.648-0.936) (Table 2). Interrater agreement of the affected side was slightly improved when patients were dichotomized (normal or abnormal findings): left side, $\kappa=0.754$, and right side, $\kappa=0.871$.

\section{Agreement on the Asymmetry of Nigrosome 1 and Clinical Laterality}

Of 24 patients with IPD, 4 showed no clinical asymmetry, whereas the other 20 patients demonstrated asymmetry (left- and rightside-dominant symptoms in 10 patients each). MR imaging showed symmetric nigrosome 1 findings in 2 patients, whereas 22 patients had asymmetric nigrosome 1 . The asymmetry in the affected nigrosome 1 at MR imaging and clinical laterality were in agreement in 19 of 24 patients $(\kappa=0.724)$. Of 20 patients with clinical asymmetry, the agreement of the asymmetry in the af- 

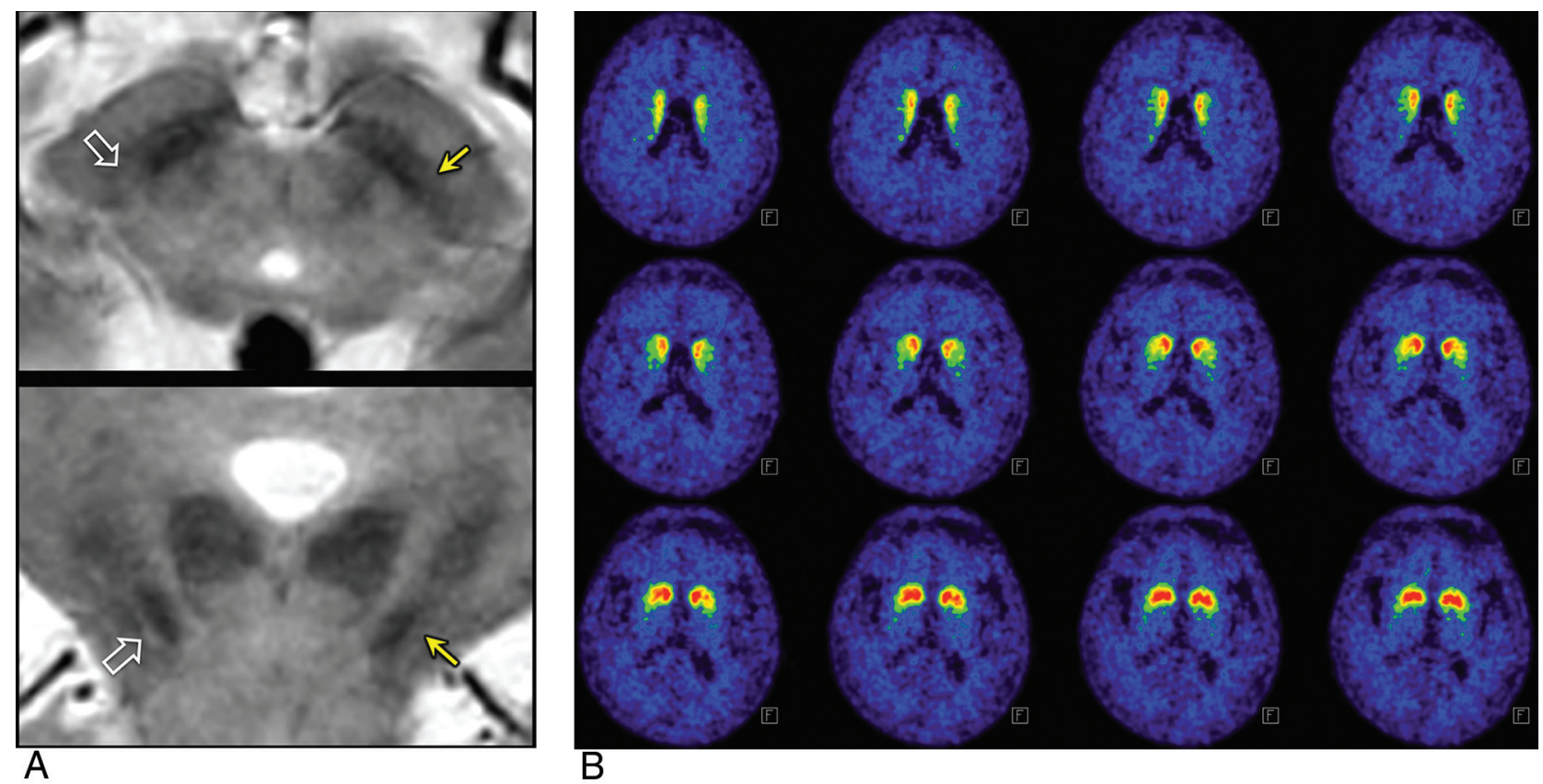

FIG 4. Asymmetry on MR imaging but not on ${ }^{18} \mathrm{~F}$-FP-CIT in a 78-year-old woman with H\&Y stage 2 and UPDRS of 4 and 2 on the right and left shows that the left nigrosome 1 is more affected (arrows) than the right one (open arrows) (A), whereas ${ }^{18} \mathrm{~F}-\mathrm{FP}-\mathrm{CIT}$ PET shows relatively symmetric findings $(B)$.

fected nigrosome 1 at MR imaging and clinical laterality increased $(\kappa=0.800)$. In the ${ }^{18} \mathrm{~F}-\mathrm{FP}-\mathrm{CIT}$ PET data, no asymmetry was observed in 10 patients. The agreement of the asymmetry on the ${ }^{18} \mathrm{~F}$-FP-CIT PET imaging and clinical laterality was fair $(\kappa=$ 0.235 ) (Fig 4). The agreement between the asymmetry in the nigrosome $1 \mathrm{MR}$ imaging and that in the ${ }^{18} \mathrm{~F}$ - FP-CIT PET imaging was fair $(\kappa=0.304)$.

\section{DISCUSSION}

Our primary findings were as follows: First, we achieved a high accuracy in the diagnosis of IPD by the detection of abnormality in nigrosome 1 at 3T MR imaging. Second, we demonstrated that the asymmetry of the affected nigrosome 1 and clinical laterality evaluated with UPDRS III had good agreement.

By using $7 \mathrm{~T}^{3-6}$ and 3T MR imaging, ${ }^{7,8}$ recent studies demonstrated that nigrosome 1 can serve as a surrogate biomarker for IPD. Schwarz et $\mathrm{al}^{7}$ obtained high-resolution 3D gradient recalled-echo imaging (voxel size, $0.55 \times 0.55 \times 0.7 \mathrm{~mm}$ ) in an imaging plane parallel to the splenium-genu line at 3T. To improve diagnostic confidence, we acquired both oblique axial and coronal images by using 3D MEDIC imaging (voxel size, $0.5 \times$ $0.5 \times 1.5 \mathrm{~mm}$ ) vertical and parallel to the longitudinal axis of the midbrain on sagittal MPRAGE, respectively. SWI is well-known for its higher sensitivity in delineating iron-containing pathology. Its SNR, however, is relatively low. We tested SWI in healthy elderly subjects before conducting this study and found that both higher SNR and higher spatial resolution are necessary to determine pathology involving nigrosome 1 . These 2 factors are conflicting in MR imaging. To address this challenge, we acquired data by using the MEDIC imaging with a relatively larger section thickness. To minimize potential complications from the thicker sections, we acquired coronal images. Using this technique, we obtained the diagnostic accuracy of $94.6 \%$. This result is between the diagnostic accuracy of a prospective case-control study (84\%) and a retrospective cross-sectional study (96\%) in the report by Schwarz et $\mathrm{al}^{7}$ and is higher than that in the study by Cosottini et al $(86 \%){ }^{8}$

It is difficult to compare the diagnostic accuracy with that in the previous studies because each study used its own imaging sequence with different image thickness (PRinciples of Echo Shifting by using a Train of Observations by Schwarz et $\mathrm{al}^{7}$ and Susceptibility-Weighted ANgiography by Cosottini et al ${ }^{8}$ ) and enrolled patients had different severities: in our study, H\&Y stage range, 1-2, UPDRS III mean, 13.5. In the prospective study by Schwarz et al, the severities were H\&Y stage 1-3, UPDRS III, $32.5^{7}$; in the study by Cosottini et al, they were $\mathrm{H} \& \mathrm{Y}$ stage $1-3$, UPDRS III, 18.3. ${ }^{8}$ Although our study subjects were in relatively earlier stages, patients with $\mathrm{H} \& \mathrm{Y}$ stage 2 were the most numerous. Thus, further studies are needed to determine the best imaging method for nigrosome 1 , with more patients with very early stages or clinical suspicion of parkinsonism.

Our second finding was that the laterality of the nigrosome 1 (more affected on either the left or right) was in concordance with the clinical laterality. This finding supports unilateral IPD corresponding to neuronal nigrostriatal degeneration in the contralateral hemisphere. As a previous study reported, abnormal findings of nigrosome 1 in IPD do not imply that the nigrosome 1 was completely lost. ${ }^{7}$ We observed that the nigrosome 1 in each hemisphere often had different appearances. This observation agrees with the finding that asymmetry of clinical features is common in the patients with IPD. Many patients have unilateral motor deficits at the time of onset of the IPD symptoms. ${ }^{14}$ Clinical asymmetry may continue in late stages of the disease, with more marked extrapyramidal symptoms on the initially affected body side. ${ }^{19}$

Although it has been reported that nigrosome 1 detection at 
3T MR imaging may serve as a surrogate biomarker for the diagnosis of IPD in previous studies, ${ }^{7,8}$ and in our study, it may still be challenging to determine the presence of subtle abnormalities of nigrosome 1 by directly looking at structures. Instead, one may compare the agreement between nigrosome 1 images and clinical laterality to improve the diagnostic confidence. To further consolidate the usefulness of the nigrosome 1 detection for the early diagnosis of IPD, however, a prospective study is needed.

In our study, the agreement on the laterality between ${ }^{18} \mathrm{~F}-\mathrm{FP}$ CIT binding and clinical symptoms was fair. This finding may suggest that ${ }^{18} \mathrm{~F}$-FP-CIT PET shows nigrostriatal functional changes that are earlier than structural changes observed in the nigrosome 1 detection and may also support the dying-back phenomenon. Alternatively, the nigrosome 1 detection might be more sensitive for neuronal degeneration. One potential complication on the laterality results is the time delay (interquartile range, 2.25-10.75 months) between the 2 imaging sessions. The earlier imaging of ${ }^{18} \mathrm{~F}$-FP-CIT PET may have resulted a lower agreement on the laterality, though the delay was relatively short considering the slow progression of the disease.

Although DAT PET or SPECT has been considered a criterion standard for the diagnosis of IPD, such imaging studies increase the medical costs and result in radiation exposure. In addition, in the very early stages, the DAT PET technique could have limitations in diagnosing Parkinson disease. A previous study reported that compensatory upregulation of presynaptic dopaminergic nerve terminals in the early stages of Parkinson disease could cause false-negative results and that dopaminergic medications might increase radiotracer uptake. ${ }^{20}$ Hence, a diagnostic marker using MR imaging (especially the more widely available 3T MR imaging rather than 7T MR imaging) will be beneficial to patients. As we have demonstrated in this study, the nigrosome 1 detection at $3 \mathrm{~T}$ MR imaging can serve as a surrogate marker for IPD in its early stage and may negate the need for ${ }^{18} \mathrm{~F}$-FP-CIT PET imaging, particularly when there is definite asymmetric abnormality in nigrosome 1 at MR imaging and the patient shows clinical laterality on the corresponding side.

A few limitations exist in this study. The number of study subjects is small. Further study with more participants will be needed to confirm the effectiveness of the nigrosome 1 detection in routine clinical diagnosis. Another limitation is a relatively large false-positive. Two participants (64 and 72 years of age) showed false-positive results. According to a previous study, nigrosome 1 was not observed in $2 \%$ of the patients without IPD older than 50 years of age, a finding that was similar to the rate of prevalence of IPD in the elderly, indicating the possibility of undiagnosed IPD. ${ }^{7}$ We could not completely exclude the possibility that the 2 healthy control subjects had presymptomatic Parkinson disease because ${ }^{18} \mathrm{~F}$-FP-CIT PET was not performed in healthy control subjects. However, no preclinical symptom such as rapid eye movement, sleep behavior disorders, anosmia, or autonomic dysfunction was observed in these subjects. An alternative explanation is that normal aging may also show changes in nigrosome 1. Further study with a large number of healthy elderly subjects is needed to elucidate this question. Last, the optimization and standardization of a sequence for the visualization of nigrosome 1 should be sought to improve diagnostic accuracy. Although we enrolled only patients with clinically well-established IPD, the specificity of our study was lower $(84.6 \%)$ than that in the studies with 7T MR imaging (87.5\%-96.2\%). ${ }^{5,6,9}$ Thus, a more specific imaging biomarker that could give confirmation to patients with clinical suspicion of parkinsonism is still needed. ${ }^{21,22}$ On the basis of our results of a relatively lower specificity than that of previous studies, nigrosome 1 detection at 3T MR imaging should be further improved to serve as a confirmatory test of IPD. The higher specificity of 7T MR imaging in the diagnosis of IPD may be due to its higher SNR and superb spatial resolution. It is difficult to improve spatial resolution substantially at $3 \mathrm{~T}$. However, we could improve the susceptibility effect without compromising SNR by susceptibility weighting of each TE image, followed by combining images afterward, which can be applicable to all 3T scanners without dependence on vendor-specific sequences. In addition, assessment of agreement on clinical laterality and imaging asymmetry may help improve specificity. With such an effort, a more specific and sensitive surrogate marker to detect the disease in the preclinical stages could be developed.

\section{CONCLUSIONS}

Our study showed that abnormality involving nigrosome 1 can be detected at 3T MR imaging with an accuracy of $94.6 \%$, and its asymmetry shows a high concordance with clinical laterality.

\section{REFERENCES}

1. Damier P, Hirsch EC, Agid Y, et al. The substantia nigra of the human brain, I: nigrosomes and the nigral matrix, a compartmental organization based on calbindin $\mathrm{D}(28 \mathrm{~K})$ immunohistochemistry. Brain 1999;122(pt 8):1421-36 CrossRef Medline

2. Damier P, Hirsch EC, Agid Y, et al. The substantia nigra of the human brain, II: patterns of loss of dopamine-containing neurons in Parkinson's disease. Brain 1999;122(pt 8):1437-48 CrossRef Medline

3. Kwon DH, Kim JM, Oh SH, et al. Seven-Tesla magnetic resonance images of the substantia nigra in Parkinson disease. Ann Neurol 2012;71:267-77 CrossRef Medline

4. Lotfipour AK, Wharton S, Schwarz ST, et al. High resolution magnetic susceptibility mapping of the substantia nigra in Parkinson's disease. J Magn Reson Imaging 2012;35:48-55 CrossRef Medline

5. Blazejewska AI, Schwarz ST, Pitiot A, et al. Visualization of nigrosome 1 and its loss in PD: pathoanatomical correlation and in vivo 7 T MRI. Neurology 2013;81:534-40 CrossRef Medline

6. Cosottini M, Frosini D, Pesaresi I, et al. MR imaging of the substantia nigra at $7 \mathrm{~T}$ enables diagnosis of Parkinson disease. Radiology 2014; 271:831-38 CrossRef Medline

7. Schwarz ST, Afzal M, Morgan PS, et al. The 'swallow tail' appearance of the healthy nigrosome: a new accurate test of Parkinson's disease-a case-control and retrospective cross-sectional MRI study at 3T. PLoS One 2014;9:e93814 CrossRef Medline

8. Cosottini M, Frosini D, Pesaresi I, et al. Comparison of 3T and 7T susceptibility-weighted angiography of the substantia nigra in diagnosing Parkinson disease. AJNR Am J Neuroradiol 2015;36: 461-66 CrossRef Medline

9. Mueller C, Pinter B, Reiter E, et al. Visualization of nigrosome 1 and its loss in PD: pathoanatomical correlation and in vivo 7T MRI. Neurology 2014;82:1752 CrossRef Medline

10. Fahn S. Description of Parkinson's disease as a clinical syndrome. Ann N Y Acad Sci 2003;991:1-14 CrossRef Medline

11. van der Hoorn A, Bartels AL, Leenders KL, et al. Handedness and dominant side of symptoms in Parkinson's disease. Parkinsonism Relat Disord 2011;17:58-60 CrossRef Medline

12. Jin S, Oh M, Oh SJ, et al. Differential diagnosis of parkinsonism 
using dual-phase F-18 FP-CIT PET imaging. Nucl Med Mol Imaging 2013;47:44-51 CrossRef Medline

13. Hughes AJ, Daniel SE, Kilford L, et al. Accuracy of clinical diagnosis of idiopathic Parkinson's disease: a clinico-pathological study of 100 cases. J Neurol Neurosurg Psychiatry 1992;55:181-84 CrossRef Medline

14. Hoehn MM, Yahr MD. Parkinsonism: onset, progression and mortality. Neurology 1967;17:427-42 CrossRef Medline

15. Scheltens $\mathrm{P}$, Barkhof $\mathrm{F}$, Leys $\mathrm{D}$, et al. A semiquantative rating scale for the assessment of signal hyperintensities on magnetic resonance imaging. J Neurol Sci 1993;114:7-12 CrossRef Medline

16. Booij J, Kemp P. Dopamine transporter imaging with [(123)I]FPCIT SPECT: potential effects of drugs. Eur J Nucl Med Mol Imaging 2008;35:424-38 CrossRef Medline

17. Wang J, Zuo CT, Jiang YP, et al. 18F-FP-CIT PET imaging and SPM analysis of dopamine transporters in Parkinson's disease in various Hoehn \& Yahr stages. J Neurol 2007;254:185-90 CrossRef Medline
18. Park E, Hwang YM, Lee CN, et al. Differential diagnosis of patients with inconclusive Parkinsonian features using [(18)F]FPCIT PET/CT. Nucl Med Mol Imaging 2014;48:106-13 CrossRef Medline

19. Kempster PA, Gibb WR, Stern GM, et al. Asymmetry of substantia nigra neuronal loss in Parkinson's disease and its relevance to the mechanism of levodopa related motor fluctuations. J Neurol Neurosurg Psychiatry 1989;52:72-76 CrossRef Medline

20. Lee CS, Samii A, Sossi V, et al. In vivo positron emission tomographic evidence for compensatory changes in presynaptic dopaminergic nerve terminals in Parkinson's disease. Ann Neurol 2000; 47:493-503 CrossRef Medline

21. Schwarz ST, Bajaj N, Gowland PA, et al. MR imaging of the substantia nigra for the diagnosis of Parkinson disease. Radiology 2014;273: 627-28 CrossRef Medline

22. Cosottini M, Frosini D, Pesaresi I, et al. Response. Radiology 2014; 273:628-29 Medline 